\title{
Two-dimensional phase-locked antiguided vertical-cavity surface-emitting laser arrays
}

\author{
D. Zhou and L. J. Mawst ${ }^{\text {a) }}$ \\ University of Wisconsin, 1415 Engineering Drive, Madison, Wisconsin 53706
}

(Received 30 June 2000; accepted for publication 22 August 2000)

\begin{abstract}
$4 \times 4$ antiguided phase-locked vertical-cavity surface-emitting laser (VCSEL) arrays have been fabricated by a selective etching process and metalorganic chemical vapor deposition regrowth. Stable, diffraction-limited output is observed corresponding to either in-phase or out-of-phase mode operation, depending on the interelement spacing width. Calculations indicate resonant leaky-wave coupling occurs for interelement spacings corresponding to an integral number of half-waves of the radiation leakage from each VCSEL region, and the use of interelement loss is effective in suppressing nonresonant modes. (C) 2000 American Institute of Physics. [S0003-6951(00)04141-3]
\end{abstract}

Coupled vertical-cavity surface-emitting laser (VCSEL) arrays are an attractive means to increase the coherent output power of VCSELs. However, stable, high-power, diffractionlimited beam operation from two-dimensional (2D) VCSEL arrays has not been realized. ${ }^{1-3}$ In addition, all previously reported phase-locked 2D VCSEL arrays operate in either an out-of-phase mode or a mixture of various modes, characteristic of weakly index guided arrays, with poor intermodal discrimination. External phase shifters have also been used on optically pumped VCSEL arrays to obtain "in-phase mode-like" emission, " however the beam is quite broad. Here we will present a novel monolithic antiguided 2D VCSEL array with stable in-phase mode emission. The progress in phase-locked 2D VCSEL arrays parallels closely that of edge-emitting phase-locked arrays. Weak coupling and poor intermodal discrimination found in evanescently coupled edge-emitting laser arrays has severely limited their single-mode output power.

By contrast, antiguided array structures exhibit strong leaky-wave coupling leading to high intermodal discrimination. As a result, edge-emitting antiguided arrays have demonstrated coherent output power in the watt range. ${ }^{6}$ The large built-in index step and strong lateral radiation leakage from an antiguide make them well suited for array integration. Antiguided VCSELs have been demonstrated by either the regrowth of high-index material in a buried heterostructure design, ${ }^{7}$ selective oxidation, ${ }^{8}$ or by a cavity-induced resonant shifted structure. ${ }^{9}$ Serkland et al. ${ }^{10}$ have recently demonstrated leaky-wave coupling between two antiguided VCSELs (coupled in-phase or out-of-phase) using structures fabricated with a cavity-induced resonance shift. Here we demonstrate that $2 \mathrm{D}(4 \times 4)$ VCSEL arrays can be designed to operate in a stable in-phase mode in good agreement with theory. Calculations show resonant coupling ${ }^{6}$ for the inphase (out-of-phase) mode occurs when the interelement spacing corresponds to an odd (even) integral number of half-waves of the antiguide radiation leakage, and that the use of interelement loss can be effective in suppressing nonresonant modes.

${ }^{a) E l e c t r o n i c ~ m a i l: ~ m a w s t @ e n g r . w i s c . e d u ~}$
For simplicity, we studied the leaky modes of a $4 \times 4$ antiguided VCSEL array structure using a fiber-mode effective-index approximation. ${ }^{11}$ These structures support leaky modes with the elements phase-locked in-phase or outof-phase, as well as intermediate modes with varying phase relationships between elements. We show that edge radiation losses and absorption losses selectively placed in the interelement regions of the array can discriminate against unwanted modes. The calculated modal edge radiation loss for the in-phase mode, $(12,12)$, and the two out-of-phase modes, $(9,9)$, and $(15,15)$, for a $4 \times 4$ array structure with 6 - $\mu$ m-wide (square) VCSELs, with an index-step of $\Delta n=0.05$ is shown in Fig. 1. The mode number convention corresponds to the number of intensity nulls of the field profile in each direction. For this structure, the in-phase mode exhibits resonant leaky-wave coupling, ${ }^{6}$ for an interelement spacing of $s=2.4$ $\mu \mathrm{m}$, since the interelement region contains an odd number of half-waves, $\lambda_{1}$, of the radiation leakage (i.e., $s=3 \lambda_{1} / 2$ ). Near the resonance condition, the in-phase mode has uniform intensity profile [Fig. 2(a)] and highest edge loss (Fig. 1), similar to that of $1 \mathrm{D}$ edge-emitting arrays. ${ }^{6}$ The out-of-phase mode [mode $(9,9)$ for $s<2.4 \mu \mathrm{m}$ ] is nonresonant and thus exhibits strong reflections in the interelement regions, resulting in high-interelement field intensity and a nonuniform (cosine-shaped) intensity profile [Fig. 2(b)].

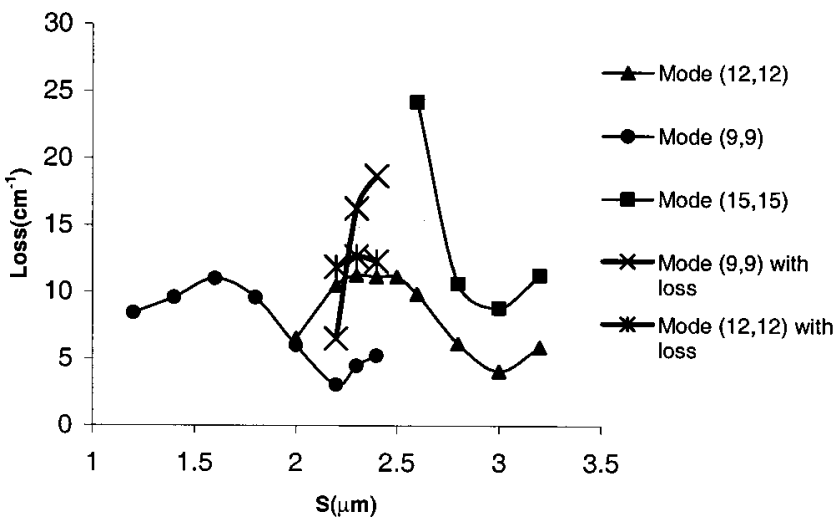

FIG. 1. Calculated modal edge radiation losses for a $4 \times 4$ VCSEL array with $d=6 \mu \mathrm{m}, \Delta n=0.05$. The effect of an interelement loss of $100 \mathrm{~cm}^{-1}$ on the in-phase mode $(12,12)$ and the lower out-of-phase mode $(9,9)$ is also shown. 


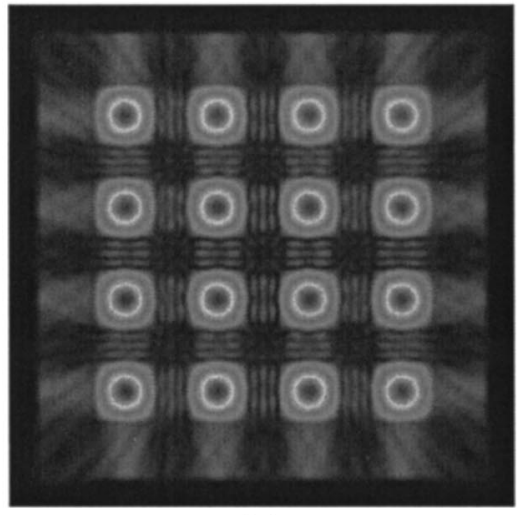

(a)

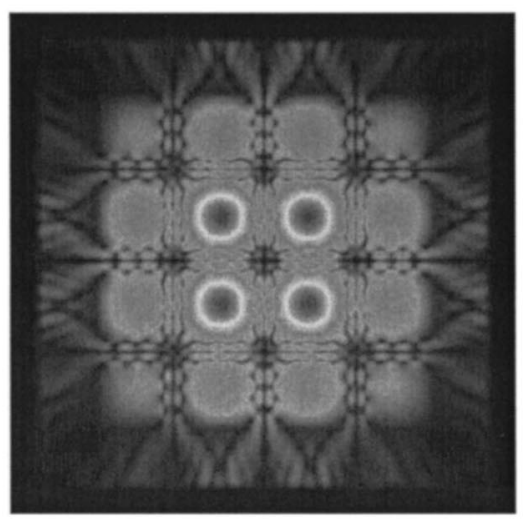

(b)

FIG. 2. Calculated intensity profiles for the (a) in-phase $(12,12)$, and (b) out-of-phase mode $(9,9)$ of $4 \times 4$ VCSEL array at $s=2.4 \mu \mathrm{m}$.

In edge-emitting antiguided arrays, the 2D gain overlap suppresses nonresonant modes, since they exhibit high field intensity in the (low gain) interelement regions. ${ }^{6}$ However, in the antiguided VCSEL array, the 2D gain overlap is not expected to be significantly mode dependent, because of the uniform gain profile. Therefore, the implementation of inter-

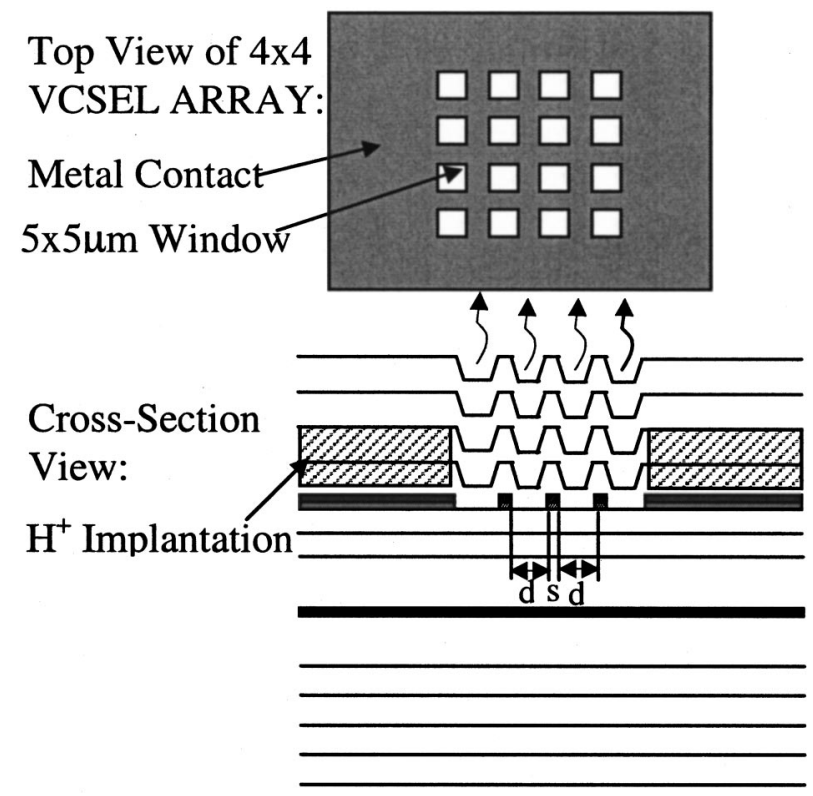

FIG. 3. Schematic diagram of a $4 \times 4$ antiguided VCSEL structure with $d=6$ $\mu \mathrm{m}$ and $s=2.5$ or $3.0 \mu \mathrm{m}$. $\mu \mathrm{m}$ and $s=2.5$ or $3.0 \mu \mathrm{m}$.

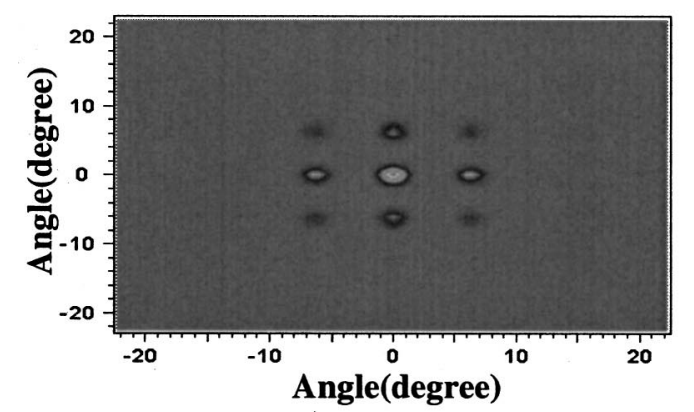

(a)

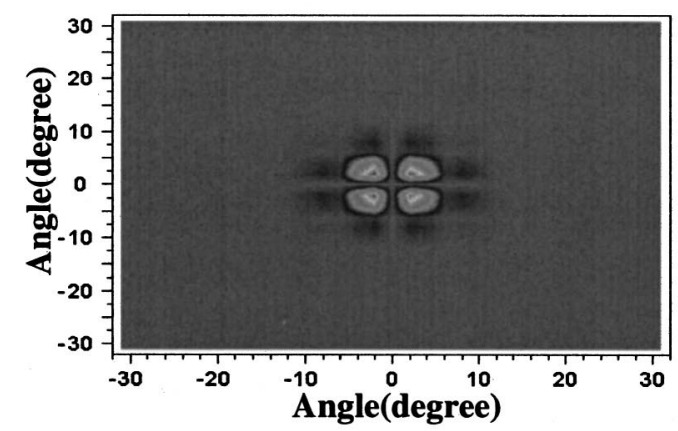

(b)

(c)

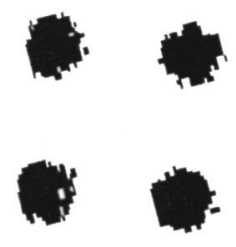

(d)

FIG. 4. Calculated far-field radiation patterns for (a) $4 \times 4$ in-phase mode, and (b) $2 \times 2$ out-of-phase mode. The measured $\mathrm{cw}$ far-field patterns for a $4 \times 4$ array operating in the (c) in-phase mode $(s=2.5 \mu \mathrm{m})$, and (d) out-ofphase $(s=3.0 \mu \mathrm{m})$ mode.

element loss into the 2D VCSEL array structure can be used to select the resonant mode, by suppressing nonresonant modes that have high field intensity in the interelement regions. This effect is demonstrated in Fig. 1, where the influence of interelement loss $\left(100 \mathrm{~cm}^{-1}\right)$ on the total modal losses for the in-phase mode $(12,12)$ and the lower out-ofphase mode $(9,9)$ is also shown. As a result, for $s>2.3 \mu \mathrm{m}$, both of the out-of-phase modes $(9,9$ and 15,15$)$ can be suppressed by either high-edge loss or interelement loss. Intermediate (adjacent) array modes are also found to have high 


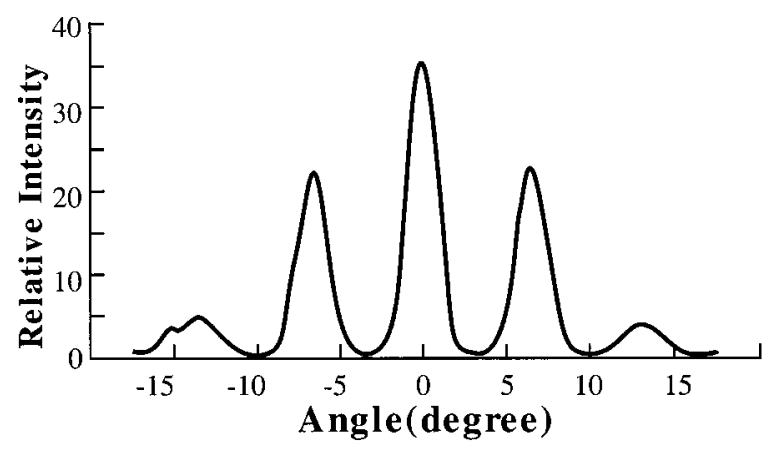

(a)

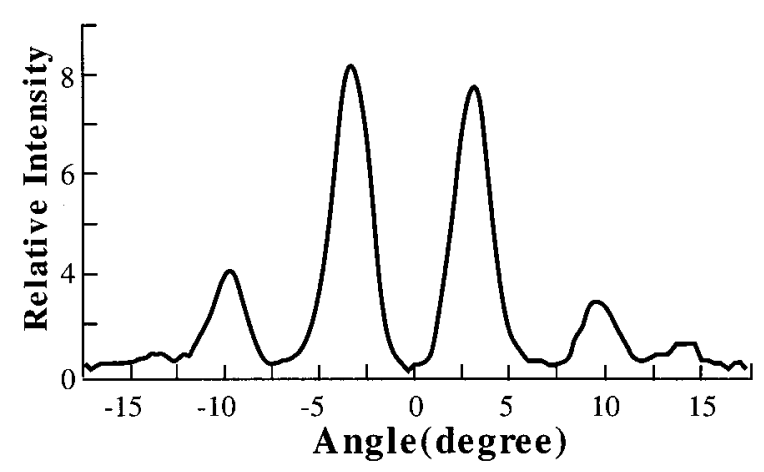

(b)

FIG. 5. 1D far-field scan at $2 \times I_{\text {th }}$ for (a) in-phase $(s=2.5 \mu \mathrm{m})$, and (b) out-of-phase $(s=3.0 \mu \mathrm{m})$ mode.

edge losses, thereby suppressing their lasing, near the inphase resonance condition. Thus, as shown in Fig. 1, a $4 \times 4$ array structure designed with interelement loss and an interelement width in the range $s=2.3-2.8 \mu \mathrm{m}$ is expected to operate in a stable in-phase array mode.

$4 \times 4$ VCSEL antiguided array structures were fabricated using a two-step MOCVD growth process ${ }^{11}$ as shown in Fig. 3 . The structure consists of 27.5 pairs of AlAs/GaAs $n$-DBR, 23 pairs of $\mathrm{Al}_{0.15} \mathrm{GaAs} / \mathrm{GaAs} p$-DBR, and $1-\lambda$ optical cavity which includes three-InGaAs quantum wells, GaAs barrier layers, and $\mathrm{Al}_{0.3} \mathrm{GaAs}$ confinement layers for $980 \mathrm{~nm}$ emission. The first growth consists of $n$-DBR, cavity, one pair of $p$-DBR and two thin spacer layers of GaAs and GaInP. ${ }^{11} \mathrm{We}$ selectively etched away these two thin layers under the emitting elements area, and then regrow the top $p$-DBR. This provides the required index step for antiguiding due to the shift of optical resonant mode in the regions containing the spacer layers. ${ }^{12}$ For the work shown here, each emitting element is a $6 \mu \mathrm{m}$ square, with an index step of $\Delta n=0.05$. The interelement (high-index region) width, $s$, was adjusted to select either the in-phase or out-of-phase resonance condition. Note that in these initial structures we do not intentionally include absorbing layers for interelement loss to aid in the suppression of nonresonant modes, as discussed above.
Arrays with interelement widths $s=2.5 \mu \mathrm{m}(s=3.0 \mu \mathrm{m})$ are observed to operate in an in-phase (out-of-phase) mode. This result is in general agreement with the calculation shown in Fig. 1, provided the modal loss curves are shifted towards higher $s$ values by $\sim 0.5 \mu \mathrm{m}$. This shift can occur due to a smaller index step $(\Delta n=0.036)$ than the design target of $\Delta n=0.05$. Since the fabricated structure does not contain interelement loss, the lasing modes observed correspond to either the nonresonant in-phase (for $s=2.5 \mu \mathrm{m}$ ) or the nonresonant out-of-phase (for $s=3.0 \mu \mathrm{m}$ ). The calculated far-field patterns for the in-phase and out-of-phase modes are shown in Figs. 4(a) and 4(b). For comparison, the measured $\mathrm{cw}$ far-field emission pattern for an array with $s=2.5 \mu \mathrm{m}$ (in-phase) and $s=3.0 \mu \mathrm{m}$ (out-of-phase), as captured by a CCD camera, are shown near laser threshold in Figs. 4(c) and 4(d). At higher driving currents, the minor side lobes become viewable, in excellent agreement with Figs. 4(a) and 4(b). The angular lobe separation and widths are obtained from a 1D scan of the far-field intensity for the two arrays, as shown in Fig. 5. The full width half maximum (FWHM) for the in-phase mode is about $2^{\circ}$, and the side lobes separation is $13.5^{\circ}$, in agreement with the calculation [Fig. 4(a)]. The emission patterns remain stable up to the maximum thermally limited $\mathrm{cw}$ output power $(\sim 1-2 \mathrm{~mW})$. Higher $\mathrm{cw}$ output powers are mainly limited due to the intensive heating for such closely packed arrays. Further optimization for high-power coherent output will be focused on how to effectively lower the device heating, such as junction down mounting, and improvements in device resistance. Finally, the optimization of lateral index-step, interelement width, and interelement loss, will be studied for obtaining the largest modal discrimination.

This work was supported by NSF Contract No. 9734283.

${ }^{1}$ J. P. Van Der Ziel, D. G. Deppe, N. Chand, G. J. Zydzik, and S. N. G. Chu, IEEE J. Quantum Electron. 26, 1873 (1990).

${ }^{2}$ M. Orenstein, E. Kapon, N. G. Stoffel, J. P. Harbison, L. T. Florez, and J. Wullert, Appl. Phys. Lett. 58, 804 (1991).

${ }^{3}$ R. A. Morgan, K. Kojima, T. Mullally, G. D. Guth, M. W. Focht, R. E. Leibenguth, and M. Asom, Appl. Phys. Lett. 61, 1160 (1992).

${ }^{4}$ M. E. Warren, P. L. Gourley, G. R. Hadley, G. A. Vawter, T. M. Brennan, B. E. Hammons, and K. L. Lear, Appl. Phys. Lett. 61, 1484 (1992).

${ }^{5}$ K.-L. Chen and S. Wang, Appl. Phys. Lett. 47, 555 (1985).

${ }^{6}$ D. Botez, L. J. Mawst, G. Peterson, and T. J. Roth, IEEE J. Quantum Electron. 26, 482 (1990).

${ }^{7}$ Y. A. Wu, G. S. Li, R. F. Nabiev, K. D. Choquette, C. Caneau, and C. J. Chang-Hasnain, IEEE J. Sel. Top. Quantum Electron. 1, 629 (1995).

${ }^{8}$ T. H. Oh, M. R. McDaniel, D. L. Huffaker, and D. G. Deppe, IEEE Photonics Technol. Lett. 10, 12 (1998).

${ }^{9}$ K. D. Choquette, G. R. Hadley, H. Q. Hou, K. M. Geib, and B. E. Hammons, Electron. Lett. 34, 1991 (1998).

${ }^{10}$ D. K. Serkland, K. D. Choquette, G. R. Hadley, K. M. Geib, and A. A. Allerman, Appl. Phys. Lett. 75, 3754 (1999).

${ }^{11}$ D. Zhou and L. J. Mawst, Appl. Phys. Lett. 76, 1659 (2000).

${ }^{12}$ G. R. Hadley, Opt. Lett. 20, 1483 (1995). 